\title{
Multiconfigurational Study of the Electronic Structure of Negatively Charged Fullerens
}

\author{
Fereshteh Naderi ${ }^{1}$ and Valera Veryazov ${ }^{2}$ \\ 1. Department of Chemistry, Shahr-e-Qods Branch, Islamic Azad University, Tehran, Iran \\ 2. Department of Theoretical Chemistry, Chemical Center, Lund, Sweden
}

\begin{abstract}
Multiconfigurational second order perturbation theory was employed in order to describe the ground and excited states of $C_{60}^{-n}$. Different choices of the active spaces are discussed and the possibility to apply multiconfigurational theory to study $C_{120}$ is investigated. The calculations were performed for all possible spin states (for selected charge) and show the preference of low spin state. The energy difference between two $C_{60}^{-3}$ and pairs $C_{60}^{-1}-C_{60}^{-5}$ and $C_{60}^{-2}-C_{60}^{-4}$ shows that the probability to create a charge alternation in fullerides is small.
\end{abstract}

Key words: Fullerenes, fullerides, multiconfigurational theory, electronic structure.

\section{Introduction}

Fullerene materials even 30 years after their discovery are under extensive theoretical study. In addition to interesting physical and chemical properties of fullerenes, such as: superconductivity [1, 2], nonlinear optics [3], ferromagnetism [4, 5] and applications in solar cells $[6,7], C_{60}$ is an interesting object to study localized electronic states, magnetic properties and metal to insulator transitions $[8,9]$. In ordinary solids, it is difficult to observe localized charged states on a long distance, but individual fullerenes are spatially isolated, thus a pair of $C_{60}$ molecules can be used to model a system with two localized spins, similar to a diatomic molecule, but on significantly larger distance between centers.

The electronic structure of crystalline fullerene is determined by weakly connected $C_{60}$ molecules. The individual $C_{60}$ molecules have rather separated in energy HOMO (highest occupied molecular orbital) with symmetry $\mathrm{H}_{\mathrm{u}}$ and LUMO (lowest unoccupied molecular orbital) $\mathrm{T}_{1 \mathrm{u}}$ with a band gap between 1.85

Corresponding author: Fereshteh Naderi, Ph.D., research field: computational study of nanostructures.
$\mathrm{eV}$ (in conductivity measurements [10]) and 2.3-2.7 $\mathrm{eV}$ (in photoemission measurements [11]).

Charge transfer fullerene compounds containing alkali atoms, fullerides, are studied much less comparing to neutral fullerens. It is known from experiment that the crystals constructed from $C_{60}$ molecules can be doped by alkali metals and form $A_{n} C_{60}$ (n = 1-6, A = Na, K, Cs) crystals. Depending on the composition and the crystal structure, these crystals can be either metals or insulators [12]. For example, a compound with the same composition $\mathrm{Cs}_{3} \mathrm{C}_{60}$ can be metallic in f.c.c. form and insulator in b.c.c. form [13].

It is known that metal to insulator transition can be caused by pairing (see, for example, Ref. [14]). In the case of fullerides such transition can correspond to the change in the electronic structure from fullerens with equivalent charge to the fullerens with alternating charge.

The negatively charged fillerene molecules are, indeed, a good molecular model for describing of the electronic structure of fullerides. A simple comparison of ionization potential of an alkali metal and electron affinity of carbon suggests the complete electron transfer from alkali metal to a fullerene molecule. 


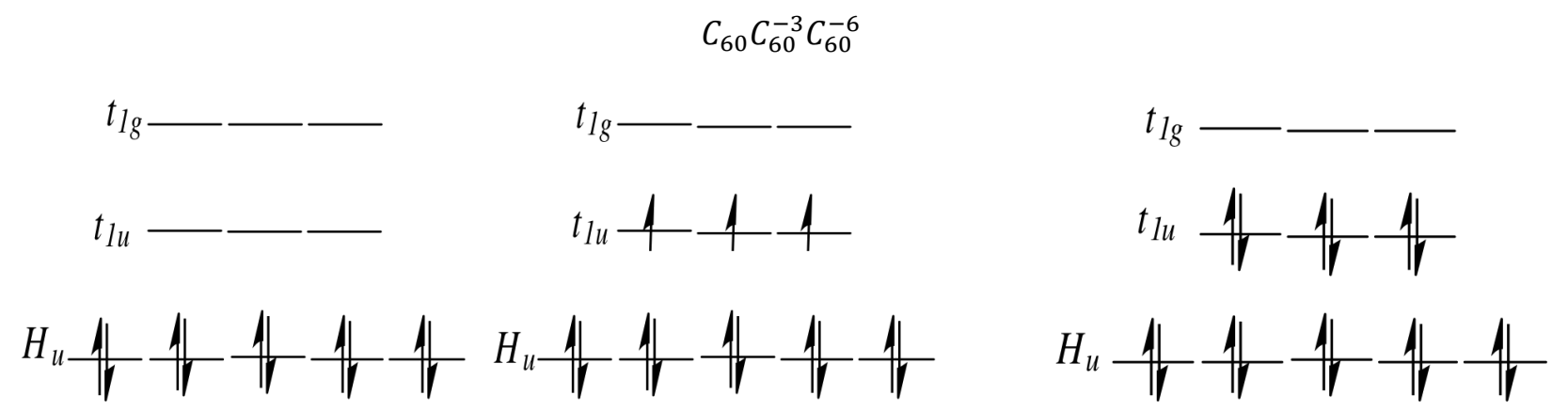

Fig. 1 Energy levels for the $C_{60}, C_{60}^{-3}, C_{60}^{-6}$ molecules.

That can be also confirmed by the results of electronic structure calculations of a system containing $C_{60}$ and $\mathrm{K}$ atom at the distances typical for fullerides. The atomic charge on alkali metal is 0.94-0.97e, which confirms the fully ionic bonding.

Fig. 1 shows the diagram of energy level for $C_{60}$, $C_{60}^{-3}, C_{60}^{-6}$ species. In $C_{60}^{-6}$ the three-fold degenerate LUMO orbitals are filled completely by the six electrons provided, for example, by the potassium ions.

In this paper, we studied the ground and excited states of $C_{60}^{-n}$ by multiconfigurational self-consistent field method MCSCF.

\section{Computational Aspects}

All calculations presented here are carried by using MOLCAS 8.0 [15] software. Since the correct description of the spin state is essential for the current study, the authors employed multiconfigurational methods: CASSCF (complete active space) or RASSCF (restrictive active space) followed by the second order perturbation theory CASPT2 and RASPT2, respectively. CASPT2 method [16] applies the second order perturbation theory to CASSCF wave function, thus the resulting wave function includes correlation effect either in exact or in approximate way. Second order perturbation theory CASPT2 can provide so-called chemical accuracy with an error less than $3 \mathrm{kcal} / \mathrm{mol}$. The basis set used in this work is Atomic Natural Orbital, ANO-S with double-zeta, VDZ, basis set (ANO-S-VDZ) [17] which has ability to describe not only the ground state but also the exited states. Increasing of the basis set to ANO-S-VDZP (including polarization functions) does not change the main trends in the calculations, but dramatically increases the computational costs. RI (resolution of identity) technique has been used for the computation of integrals.

In order to avoid unphysical symmetry constrains of the solution for the wave function, the symmetry has not been enforced in our calculations. In the same time, the obtained solutions preserved the icosahedral symmetry.

In $C_{60}$ the highest occupied molecular orbital, HOMO, is a five fold degenerate: $\mathrm{H}_{\mathrm{u}}$, and the LUMO and LUMO +1 orbitals are two three-fold degenerate representations: $T_{1 u}$ and $T_{2 u}$. A selection of the active space, which in general is a rather complicated task [18], in case of $C_{60}$ turns to a straight forward procedure. The orbitals near HOMO-LUMO gap are isolated by energy, and their high degeneracy does not leave many choices. The authors made a set of test calculations, varying the number of orbitals included into the active space in CASSCF, but the optimal (in terms of the total energy and the time of the calculation) result was obtained for 8 active orbitals (HOMO and LUMO) and $10+$ n electrons for $C_{60}^{-n}$.

The authors should note here that to study two $C_{60}$ molecules one has to double the active space. A test calculation of $C_{120}$ molecule with ANO-S-VDZ basis set and 22 orbitals treated within RASPT2 approach requires a computer with at least $128 \mathrm{~Gb}$ RAM and at least $1 \mathrm{~Tb}$ hard drive. $C_{120}$ is a challenging molecule 
even for modern methods, like DMRG approach [19] due to the combination of the large active space and the large basis set located at different centers, thus the reduction of the computational cost due to resolution of identity is moderate.

For the selection of the active space the authors employed RASSCF technique, selecting so-called RAS1 space for the orbitals with up to a maximum number of holes (usually made by single and double excitations), RAS2 space for the complete set of excitations, and finally RAS3 space for the orbitals which can be occupied by a maximum number of electrons (usually by single and double excitations). The authors will use symbol $/ 1 / \mathrm{m} / \mathrm{n} /$ to indicate the number of orbitals in RAS1 (1), RAS2 (m), and RAS3 (n). In this work RASSCF calculations are carried out through $\mathrm{H}_{\mathrm{u}}, \mathrm{T}_{1 \mathrm{u}}$ and $\mathrm{T}_{2 \mathrm{u}}$ orbitals. $/ 5 / 3 / 3 /$ is a symbol for RASSCF calculation having 5 orbitals in the RAS1 space, 3 orbitals in RAS2 and 3 orbital in RAS3 space. Two holes in RAS1 and two electrons in RAS3 are allowed.

\section{Results and Discussion}

Because of the large number of $\pi$-electrons in $C_{60}$, it has a capacity to create negatively charged fullerenes up to $C_{60}^{-6}$. The energetical stability of these anions and the spin states is the subject of this study. Since in the bulk fullerenes (and fullerites) the individual $C_{60}$ molecules are spatially separated, $C_{60}^{-n}$ anion is a good model system to describe the properties of the crystal.

Depending on the atomic charge on fullerene, different spin states are possible. Table 1 shows different charges and multiplicities of structures that are studied. Here after the authors call parallel $(\mathrm{P})$ and antiparallel (aP) spin state for parallel and antiparallel spin configuration, respectively. These extra charges can be distributed on electron clouds around each carbon atom, on h-h (hexagon-hexagon bond) or h-p (hexagon-pentagon bond). Fig. 2 illustrates that extra electrons are not delocalized over all bonds. It seems each additional electron starts to go just on (h-p) bonds. Finally six additional electrons create pentagon clouds on each pentagon. Finally in $C_{60}^{-6}$, six extra electrons form pentagon clouds on each pentagon. $C_{60}^{-n}$ is in the most symmetrical structure where $\mathrm{n}$ is 0 , -6 or -3 with quadruplet multiplicity. In Fig. 2 this symmetry can be seen.

In negatively charged $C_{60}$ the extra electrons are delocalized and they can be correlated by other electrons. Hence the basis set is very important in these kinds of ions. For instance Wierzbowska et al. calculated the IP (ionization potential) for the -1 charged $C_{60}$, using the 6-31 Gaussian basis set [6s3sp1sp]/[1s2sp] at $0.633 \mathrm{eV}$ [20] which is obviously different from the experimental $2.65 \pm 0.05$ $\mathrm{eV}$ [21]. They continued their calculations performing with the minimal basis set of order 3-21 $([6 s 3 p] /[2 s 1 p])$, which also allowed for a (restricted open shell Møller-Plesset) ROMP2 calculation, to check the effects of the basis set and of additional correlations. The IPs was still so far from experimental values. Although, Lezius et al. are not sure about experimental values [22], a comparison of previous DFT calculations on IP by Rosen and Wastberg in 1989 [23], de Coulon et al. in 1992 [24],

Table 1 Different charges and multiplicities of $C_{60}^{-n}(0 \leq \mathrm{n} \leq 6)$ and differences in energies (eV).

\begin{tabular}{|c|c|c|c|c|c|c|c|c|c|c|}
\hline Charge & 0 & -1 & & -2 & & -3 & & -4 & -5 & -6 \\
\hline Spin state & 1 & 2 & 1 & 3 & 2 & 4 & 1 & 3 & 2 & 1 \\
\hline$\Delta \mathrm{E}_{1}^{[\mathrm{a}]}$ & 0 & -2.35 & -2.17 & -1.79 & +1.29 & +1.53 & +7.66 & +7.73 & +16.27 & +27.66 \\
\hline$\Delta \mathrm{E}_{2}^{[\mathrm{b}]}$ & 0 & -3.22 & -2.12 & -1.71 & +1.46 & +1.86 & +7.51 & +7.87 & +17.09 & +29.03 \\
\hline$\Delta \mathrm{E}_{3}{ }^{[\mathrm{c}]}$ & -0.22 & -0.23 & -0.27 & -0.30 & -0.40 & -0.55 & -0.07 & -0.35 & -1.04 & -1.59 \\
\hline
\end{tabular}

[a] $\Delta \mathrm{E}_{1}=\mathrm{E}_{/ 0 / 8 / 0 /} C_{60}^{-n}-\mathrm{E}_{/ 0 / 8 / 0 /} C_{60}$, where $\mathrm{E}_{/ 0 / 8 / 0 /}$ is the energy that calculated for $/ 0 / 8 / 0 /$ active space.

[b] $\Delta \mathrm{E}_{2}=\mathrm{E}_{/ 5 / 3 / 3} / C_{60}^{-n}-\mathrm{E}_{/ 5 / 3 / 3 / 3} C_{60}$, where $\mathrm{E}_{/ 5 / 3 / 3 / 3}$ is the energy that calculated for $/ 5 / 3 / 3 /$ active space.

$[\mathrm{c}] \Delta \mathrm{E}_{3}=\mathrm{E}_{/ 0 / 8 / 0 /} C_{60}^{-n}-\mathrm{E}_{/ 5 / 3 / 3 /} C_{60}^{-n}$. 


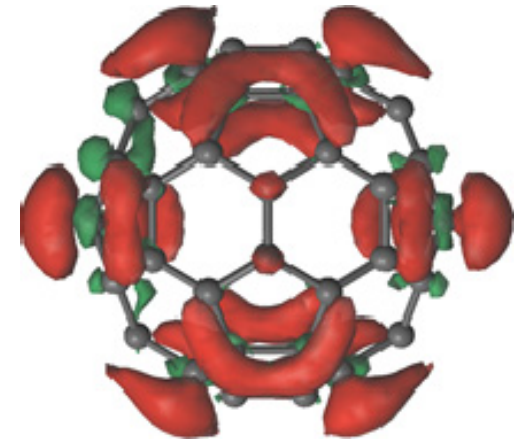

(a)

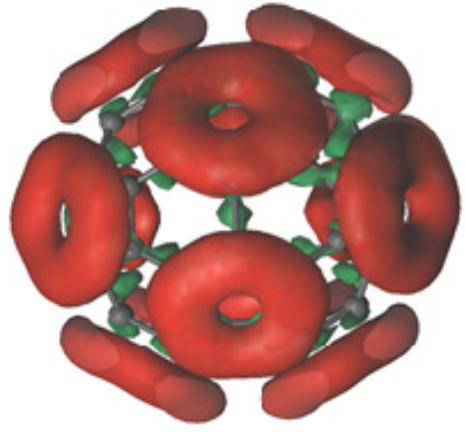

(b)

Fig. 2 Differences of charge densities between (a) $C_{60}^{-3}$ and $C_{60}$, (b) $C_{60}^{-6}$ and $C_{60}$.

Razafinjanahary et al. in 1994 [35] and Wierzbowska et al. in 2004 [20] have shown the importance of exchange-correlation function. Because of sensitivity to polarization and to correlations of extra electrons in fullerene and importance of choosing basis set, DFT calculations are not suitable for negatively charged $C_{60}$ which are strongly correlated systems. 2 and 4 negatively charged fullerenes have an insulating and diamagnetic character [26-28] while there is a metallic and even superconductivity character in 3 negatively charged fullerenes [29-31]. Despite this knowledge a full understanding of metallic behavior of fullerides is still lacking.

To consider dynamic correlation and second order perturbation theory, the authors use CASPT2 and RASPT2 methods in our calculations. When the numbers of electrons increase, the relative energies go to positive side indicating that the addition of an electron decreases the stability of the anions in both $/ 0 / 8 / 0 /$ and $/ 5 / 3 / 3 /$ selected active space. Also differences in energies of each charge and multiplicity (spin) between two selected active spaces are represented in Table 1 . As expected the species are more stable when /5/3/3/ active space is selected. These differences show the importance of choosing the active space. More details are shown in Table 1.

In Table 2, to investigate the symmetry of species, the authors compare the energies in pairs. For instance the average of energies in $C_{60}^{-1}$ and $C_{60}^{-5}$ indicates that this average is much more than the average of energies in two $C_{60}^{-3}$ with spin state 2 and 4 . This table shows more stability of two $C_{60}^{-3}$ vs. $C_{60}^{-2}$ and $C_{60}^{-4}$ with triplet and singlet spin state.

Table 3 indicates the variations of energies with a change in spin state when the charge is the same. In each charge, antiparallel spin state is favorable. It is the same in both selected active space. Due to the results obtained of the model Hamiltonian which was reported in 29, spread of the multiplets for all negatively charged states of $C_{60}$ is about $0.6 \mathrm{eV}$. The authors find that these energies are about $0.4 \mathrm{eV}$ in /5/3/3/ active space as shown in Table 3. As mentioned in 29, weakly bound electron states in negative ions are not only sensitive to polarization and to correlation but also to basis sets.

Experimental evidences proved this matter. Metalic $C_{60}^{-3}$, antiparallel state, is more stable in normal state than super conductive $C_{60}^{-3}$ which is face-centered cubic structures with three half-filled molecular orbitals [32]. There is a competition to occupy less energetic levels among the different states.

Modeling of the bulk properties of fullerides by a separate fullerene molecule is an approximation. The distance between fullerene surfaces in the bulk material is close to $3 \AA$, so they can interact, and in particular the electron transfer could take place. Thus the ultimate test would include a calculation which involves two fullerenes on a short distance. Such calculation involves the decision about mutual orientation of the molecules. DFT calculations of two $C_{60}$ on the distance $10 \AA$ between the centers (which is very close to the experimental value) shows very 
Table 2 Comparison of average of energies in different species of $C_{60}^{-n}$ in $-1,-5$ and $-2,-4$ and $-3,-3$ charge and multiplicities. Energies are in $\mathrm{eV}$. These energies are calculated in $/ 5 / 3 / 3 /$ active space.

\begin{tabular}{llll}
\hline Species & Spin state & $\mathrm{E}$ & $\mathrm{E}_{\mathrm{Av} .}$ \\
\hline$C_{60}^{-1}$ & 2 & $-61,934.51$ & $-61,924.80$ \\
$C_{60}^{-5}$ & 2 & $-61,915.09$ & \\
$C_{60}^{-2}$ & 3 & $-61,933.88$ & $-61,929.09$ \\
$C_{60}^{-4}$ & 3 & $-61,924.30$ & \\
$C_{60}^{-2}$ & 1 & $-61,934.30$ & $-61,929.48$ \\
$C_{60}^{-4}$ & 1 & $-61,924.66$ & \\
$C_{60}^{-3}$ & 2 & -61930.72 & $-61,930.52$ \\
$C_{60}^{-3}$ & 4 & -61930.31 & \\
\hline
\end{tabular}

Table 3 Differences of energies $(\mathrm{eV})$ for $C_{60}^{-n}(1 \leq \mathrm{n} \leq 6)$.

\begin{tabular}{llllll}
\hline $\mathrm{n}$ & $\Delta E_{a P P}^{n}[\mathrm{a}]$ & $\Delta E_{a P P}^{m}[\mathrm{~b}]$ & $\mathrm{n}$ & $\Delta E_{a P P}^{n}$ & $\Delta E_{a P P}^{m}$ \\
\hline-1 & - & - & -4 & +0.08 & +0.36 \\
-2 & +0.38 & +0.42 & -5 & - & - \\
-3 & +0.25 & +0.40 & -6 & - & - \\
\hline
\end{tabular}

[a], [b] $\Delta E_{a P P}^{n}$ and $\Delta E_{a P P}^{m}$ indicate variation of the energy due to transition of the system from an antiparallel state of charge $\mathrm{n}$ to a parallel state of charge $n$ in $/ 0 / 8 / 0 /$ and $/ 5 / 3 / 3 /$ active space, respectively.

small changes in the total energy dependently on rotation of fullerene and the direction of the bonds. As the authors found, the most stable structure was obtained when the angle between to $\mathrm{h}-\mathrm{h}$ bond in two $C_{60}$ was 90 degree. The energy gain for this geometry is $0.8 \mathrm{kcal} / \mathrm{mol}$.

Performing the calculation on the same level of theory as for $C_{60}$ is a challenging task, since the active space is doubled. However, the division of the active space between RAS1 and RAS2 makes possible to use 22 active orbitals in total (with $10 \mathrm{RAS} 1 / \mathrm{SD}$ and 12 RAS2 orbitals). The total energy $C_{120}$ in comparison to double value of $C_{60}$ deviates in the order of 0.5 to $1.5 \mathrm{eV}$ for neutral fullerenes and with charge -2 , correspondingly. This difference suggests that the study of the electronic structure of $C_{120}$ is desired.

\section{Conclusions}

The authors presented calculations of negatively charged fullerenes performed within multiconfigurational approach, and analyzed the total energies of different spin states. The active space which includes at least 11 active orbitals for each fullerene molecule is needed. For all negative charges, low spin configuration is preferable. Our calculations also demonstrate that equally distributed charge (between fullerenes) is energetically favorable, i.e. two $C_{60}^{-3}$ have lover energy comparing to any other pair of fullerenes with total charge $-6\left(C_{60}^{-1}-C_{60}^{-5}\right.$ and $\left.C_{60}^{-2}-C_{60}^{-4}\right)$.

\section{Acknowledgments}

The Swedish National Infrastructure for Computing (SNIC) through Lunarc, the Center for Scientific and Technical Computing at Lund University.

\section{References}

[1] Takeya, H., Miyazawa, K., Kato, R., Wakahara, T., Ozaki, T., Okazaki, H., Yamaguchi, T., and Takano, Y. 2012. "Superconducting Fullerene Nanowhiskers." Molecules 17: 4851-9.

[2] Hebard, A., Rosseinsky, M., Haddon, R., Murphy, D., Glarum, S. H., Palstra, T. T. M., Ramirez, A. P., and Kortan, A. R. 1991. "Superconductivity at $18 \mathrm{~K}$ in Potassium-doped $\mathrm{C}_{60}$." Nature 350: 600-1.

[3] Sheka, E. 2011. "Nanophotonics of Fullerene. 2. Linear and Non-Linear Optics." Nanosci. Nanotechnol. Lett. 3: 34-40.

[4] Ren, S., Wuttig, M., 2012. "Organic Exciton Multiferroics. Advanced Materials.” Advanced Materials 
24: 724-7.

[5] Allemand, P. M., Khemani, K. C., Koch, A., Wudl, F., Holczer, K., Donovan, S., Gruner, G., Thompson, J. D. 1991. "Organic Molecular Soft Ferromagnetism in a FULLERENE C 60 . " Science 253: 301-2.

[6] Wilken, S., Scheunemann, D., Wilkens, V., Parisi, J., and Borchert, H. 2012. "Improvement of ITO-free Inverted Polymer-based Solar Cells by Using Colloidal Zinc Oxide Nanocrystals as Electron-Selective Buffer Layer." Org. Electron. 13: 2386-94.

[7] Li, C. Z., Yip, H. L., and Jen, A. K. Y. 2012. "Functional Fullerenes for Organic Photovoltaics." Journal of Materials Chemistry 22: 4161-77.

[8] Durand, P., Darling, G., Dubitsky, Y., Zaopo, A., and Rosseinsky, M., 2003. "The Mott-Hubbard Insulating State and Orbital Degeneracy in the Superconducting $C_{60}^{-3}$ Fulleride Family." Nat. Mater. 2: 605-10.

[9] Takabayashi, Y., Ganin, A. Y., Jeglic, P., Arcon, D., Takano, T., Iwasa, Y., Ohishi, Y., Takata, M., Takeshita, N., Prassides, K., and Rosseinsky, M. J. 2009. "The Disorder-free Non-BCS Superconductor $\mathrm{Cs}_{3} \mathrm{C}_{60}$ Emerges from an Antiferromagnetic Insulator Parent State." Science 323: 1585-90.

[10] Rabenau, T., Simon, A., Kremer, R. K., and Sohmen, E. 1993. "The Energy Gaps of Fullerene $C_{60}$ and $C_{70}$ Determined from the Temperature Dependent Microwave Conductivity." Phys. B. 90: 69-72.

[11] Benning, P. J., Martins, J. L., Weaver, J. H., Chibante, L. P. F., and Smalley, R. E. 1991. "Electronic States of $\mathrm{K}_{\mathrm{x}} \mathrm{C}_{60}$ : Insulating, Metallic, and Superconducting Character." Science 252: 1417-21.

[12] Käämbre, T., Schnadt, J., Norlund, D., Glover, C. J., Rubensson, J. E., Rudolf, P., Mårtensson, N., Nordgren, J., and Bruhwiler, P. A. 2007. "Bulk Electronic Structure of $\mathrm{K}_{3} \mathrm{C}_{60}$ as Revealed by Soft X-rays." Phys. Rev. B Solid State 75: 195432.

[13] Ganin, A. Y., Takabayashi, Y., Jeglic, P., Arcon, D., Potočnik, A., Baker, P. J., Ohisi, Y., McDonald, M. T., Tzirakis, M. D., McLennan, A., Darling, G. R., Takata, M., Rosseinsky, M. J., and Prassides, K. 2010. "Polymorphism Control of Superconductivity and Magnetism in $\mathrm{Cs}_{3} \mathrm{C}_{60}$ Close to the Mott Transition." Nature 466: 221-5.

[14] Neaton, J. B., and Ashcroft, N. W. 1999. "Pairing in Dense Lithiumnature." Nature 400 (6740): 141-4.

[15] Aquilante, F., Autschbach, J., Carlson, R. K., Chibotaru, L. F., Delcey, M. G., De Vico, L., Fdez. Galván, I., Ferré, N., and et al. 2016. "Molcas 8: New Capabilities for Multiconfigurational Quantum Chemical Calculations Across the Periodic Table." J. Comput. Chem. 37: 506-41.

[16] Andersson, K., Malmqvist, P. Å, and Roos, B. O. 1992.
"Second-Order Perturbation Theory with a Complete Active Space Self-consistent Field Reference Function." J. Chem. Phys. 96: 1218-26.

[17] Pierloot, K., Dumez, B., Widmark, P. O., and Roos, B. O. 1995. "Density Matrix Averaged Atomic Narural Orbital (ANO) Basis Sets for Correlated Molecular Wave Functions. IV. Medium Size Basis Sets for the Atoms H-Kr." Theor. Chim. Acta. 90: 87-114.

[18] Veryazov, V., Malmqvist, P. Å., and Roos, B. O. 2011. "How to Select Active Space for Multiconfigurational Quantum Chemistry?.” Int. J. Quantum Chem. 111 (111): 3329-38.

[19] Schollwoeck, U., 2011. "The Density-Matrix Renormalization Group in the Age of Matrix Product States." Ann. Phys.326: 96-192.

[20] Wierzbowska, M., Lüders, M., and Tosatti, E. 2004. "Multiplet Structures of Charged Fullerenes." J. Phys. B: At., Mol. Opt. Phys. 37: 2685-98.

[21] Yang, S., Pettiette, C., Conceicao, J., Cheshnovsky, O., and Smalley, R. 1987. "UPS of Buckminsterfullerene and Other Large Clusters of Carbon.” Chem. Phys. Lett. 139: 233-8.

[22] Lezius, M., Scheier, P., and Märk, T. 1993. "Free Electron Attachment to $\mathrm{C}_{60}$ and $\mathrm{C}_{70}$." Chem. Phys. Lett. 203: 232-6.

[23] Rosén, A., and Wästberg, B. 1989. "Calculations of the Ionization Thresholds and Electron Affinities of the Neutral, Positively and Negatively Charged $\mathrm{C}_{60}$-Follene-60." J. Chem. Phys. 90: 2525-6.

[24] Coulon, V., Martins, J., and Reuse, F. 1992. "Electronic Structure of Neutral and Charged $\mathrm{C}_{60}$ Clusters." Phys. Rev. B. 45: 13671-5.

[25] Razafinjanahary, H., Rogemond, F., and Chermette, H. 1994. "Incidence of the Muffin-tin Approximation on the Electronic Structure of Large Clusters Calculated by the MS-LSD Method: The Typical Case of $\mathrm{C}_{60}$." Int. J. Quantum Chem. 51: 319-28.

[26] Fabrizio, M., and Tosatti, E. 1997. "Nonmagnetic Molecular Jahn-Teller Mott Insulators.” Phys. Rev. B. 55: 13465.

[27] Knupfer, M., and Fink, J. 1997. "Mott-Hubbard-like Behavior of the Energy Gap of $\mathrm{A}_{4} \mathrm{C}_{60}(\mathrm{~A}=\mathrm{Na}, \mathrm{K}, \mathrm{Rb}, \mathrm{Cs})$ and $\mathrm{Na}_{10} \mathrm{C}_{60} . "$ J. Phys. Rev. Lett. 79: 2714-7.

[28] Chibotaru, L., Ceulemans, A., and Cojocaru, S. 1999. "Electronic Structure of $\mathrm{A}_{4} \mathrm{C}_{60}$ : Joint Effect of Electron Correlation and Vibronic Interactions." Phys. Rev. B. 59: R12728.

[29] Kiefl, R. F., Duty, T., Schneider, J., MacFarlane, A., Chow, K., Elzey, J., Mendels, P., Morris, G. D., and et al. 1992. "Evidence for Endohedral Muonium in $\mathrm{K}_{\mathrm{x}} \mathrm{C}_{60}$ and Consequences for Electronic Structure." Phys. Rev. lett. 69: 2005-8. 
[30] Kosaka, M., Tanigaki, K., Hirosawa, I., Shimakawa, Y., Kuroshima, S., Ebbesen, T., Mizuki, J., and Kubo, Y. 1993. "ESR Studies of K-doped $\mathrm{C}_{60}$." Chem. Phys. lett. 203: 429-32.

[31] Stepniak, F., Benning, P., Poirier, D., and Weaver, J. 1993. "Electrical Transport in $\mathrm{Na}, \mathrm{K}, \mathrm{Rb}$, and $\mathrm{Cs}$
Fullerides: Phase Formation, Microstructure, and Metallicity.” J. Phys. Rev. B. 48: 1899-906.

[32] Mitrano, M., Cantaluppi, A., Nicoletti, D., Kaiser, S., Perucchi, A., Lupi, S., Di Pietro, P., Pontiroli, D., and et al. 2015. "An Optically Stimulated Superconducting-like Phase in k3c60 far above Equilibrium tc." Physics. 